\title{
Preventing and Managing Hydration and Dehydration in Older People
}

\author{
Vilborg Kolbrún Vilmundardóttir \\ and Sigrún Sunna Skúladóttir
}

\begin{abstract}
Previous chapters have described nutritional care in geriatrics and orthogeriatrics in detail, including special focus on malnutrition and best practice in nursing care. This chapter will focus on recommendations and guidelines for hydration, fluid intake and intravenous fluid therapy in geriatrics and orthogeriatrics.
\end{abstract}

\section{Keywords}

Fluid therapy - Water-electrolyte balance - Dehydration · Practice guidelines · Older adults

This chapter is a component of Part I: Nutritional Care in Old Age.

For an explanation of the grouping of chapters in this book, please see Chap. 1: 'Overview of Nutrition Care in Geriatrics and Orthogeriatrics'.

\footnotetext{
V. K. Vilmundardóttir $(\bowtie)$

The Faculty of Food Science and Nutrition, School of Health Sciences, University of Iceland, Reykjavik, Iceland e-mail:vkv2@hi.is

S. S. Skúladóttir

The Faculty of Food Science and Nutrition, School of Health Sciences, University of Iceland, Reykjavik, Iceland

The Faculty of Nurse, School of Health Sciences, University of Iceland, Reykjavik, Iceland e-mail: sss37@hi.is
} 


\section{Learning Outcomes}

By the end of this chapter, you will be able to:

- Recognise and apply evidence-based recommendations and guidelines for hydration, fluid intake and intravenous fluid therapy for geriatric and orthogeriatric patients in hospital and outpatient settings, residential aged care and community living.

- Recognise age-related changes that influence hydration and fluid intake in older adults.

- Distinguish between the two subcategories of dehydration (low-intake dehydration and volume depletion).

- Implement best practice to screen for, assess and reassess dehydration and fluid balance at all stages in hospital.

- Implement best practice to treat dehydration and other hydration disturbances at all stages in hospital.

\subsection{Geriatric Orthopaedic Patients}

Geriatric orthopaedic patients are an especially frail and vulnerable group, considered at risk of being both dehydrated and malnourished [1-3]. Dehydration can have adverse health outcomes [4-7] (Table 7.1).

Adverse health outcomes (Table 7.1) can increase hospital-acquired complications and adverse events as well as the risk for readmission to hospital [4, 7]. This in turn can contribute to adverse outcomes and increased healthcare costs $[4,7,8]$.

\subsection{Hydration in Geriatrics}

Proper hydration, where fluid intake and fluid losses are balanced, is a crucial component in maintaining bodily functions [9]. Hence, ensuring fluid intake that accounts for losses via urine, faeces, evaporation (lung and skin), wounds, surgery and medical procedures is essential. Fluid requirements are individualised, mainly based on caloric consumption, water losses and kidney capacity [6, 9]. In all settings, providing adequate amounts of fluid with drinks, nasogastrically,

Table 7.1 Adverse health outcomes commonly associated with dehydration

\footnotetext{
- Confusion

- Constipation

- Decreased quality of life

- Delirium

- Disability

- Drug toxicity

- Falls and fractures

- Heart disease
}

- Infections

- Longer stay in hospital

- Morbidity and mortality

- Pain

- Pressure ulcers

- Renal failure

- Urinary tract infection 
subcutaneously or intravenously to prevent dehydration whilst averting excessive amounts should be considered a priority.

\subsubsection{Recommendations for Older Adults}

Generally, an adequate intake (AI) of fluid for older adults is defined as $2.0 \mathrm{~L} /$ day for women and $2.5 \mathrm{~L} /$ day for men [6, 9]. Assuming $20 \%$ of fluids come from eating foods, this means women need at least $1.6 \mathrm{~L} /$ day of drinks and men $2.0 \mathrm{~L} /$ day. Clinical situations and environmental factors (i.e. higher temperatures) can change individual fluid needs, and in some patient groups, fluid restriction might be needed, e.g. those with heart failure or renal failure $[5,6]$. For older adults that possibly need fluid restriction, an interdisciplinary approach is crucial to configure fluid requirements and timeframes of restrictions. It is important that older adults and interdisciplinary healthcare providers are able to identify fluids in both drinks and also foods (Table 7.2).

Drinks for older adults should include a range of different options, based on individual preferences [6]. There are many diverse drinks that provide fluids to the body, ranging from water, juices, milk-based supplements, smoothies, sports drinks, soups, tea or coffee and alcoholic beverages.

Offering a range of preferred drinks frequently during the day can improve total fluid intake and thus minimise the risk of dehydration [6]. Those at risk of malnutrition or malnourished should be offered energy- and/or protein-dense drinks, e.g. milk, milk-based drinks, smoothies or oral nutritional support (Chap. 5). Energyand protein-dense drinks provide fluid as well as being an important source of energy, protein and nutrients. It is worthy to note that tea and coffee are both sources of fluid, but older patients should be encouraged to include other drinks throughout the day, thus possibly providing energy, protein and nutrients.

\subsubsection{Age-Related Changes Associated with Hydration}

Research indicates lower fluid intakes in older adults compared to younger groups, with different and interrelated causes $[6,8,9]$. Lower intakes are possibly explained

Table 7.2 Examples of amounts of fluid found in common drinks/foods

\begin{tabular}{|l|l|l|}
\hline Food product & Portion size & Fluid $(\mathrm{mL})$ \\
\hline Whole milk $(3.9 \%)$ & 1 cup $(250 \mathrm{~mL})$ & 220 \\
\hline Coffee/tea & 1 cup $(250 \mathrm{~mL})$ & 250 \\
\hline Apple juice & 1 cup $(250 \mathrm{~mL})$ & 220 \\
\hline Porridge & 1 bowl $(200 \mathrm{~g})$ & 180 \\
\hline Oral nutritional supplement drink & 1 bottle $(200 \mathrm{~mL})$ & 140 \\
\hline Yogurt & 1 tub $(200 \mathrm{~g})$ & 180 \\
\hline Boiled pasta/rice & 1 bowl $(200 \mathrm{~g})$ & 140 \\
\hline Boiled potatoes & 3 small sized $(150 \mathrm{~g})$ & 120
\end{tabular}


by physiological changes that occur with higher age, including decreases in kidney capacity and sense of thirst $[6,8]$. Furthermore, total body water reduces with higher age, resulting in smaller fluid reserves. Medications also need consideration in this context, as many commonly used medications among older adults can encourage water losses, including diuretics and laxatives. Other potential causes include choosing to reduce drinking due to fear of incontinence and problems with getting to the bathroom; memory problems, i.e. forgetting to drink; social isolation; physical access to drinks; and problems with swallowing. Thus, all older adults are considered at risk of being dehydrated, needing special attention and encouragement when it comes to fluid intake $[6,9]$.

\subsection{Dehydration and Other Hydration Disturbances}

Undoubtedly, the main concern regarding hydration in older adults is dehydration, a commonly encountered problem in clinical situations, with reported prevalence among older adults in hospitals ranging from $4 \%$ to $58 \%$ [6].

Diagnosing dehydration is complex, due to differences in definitions and a wide range of possible causes $[4,6]$. Generally, dehydration can be defined into two subcategories, based on whether a shortage of water is caused by low intake or excessive loss (Table 7.3).

Dehydration is associated with adverse outcomes in older adults, including increased morbidity and mortality, longer stay in hospital and increased risk of disability $[4,6]$. Early diagnosis of dehydration in clinical settings is crucial, allowing for timely interventions and possibly prevention. Screening for low-intake dehydration and volume depletion (volume status) is recommended for all geriatric patients upon admission into hospital (Fig. 7.1).

\subsubsection{Screening and Assessment of Dehydration}

Underlying causes of dehydration can vary greatly, making screening and assessment in older adults complex $[4,6]$. First and foremost assessment (and reassessment) should include detailed information on $[4,5]$ :

Table 7.3 Dehydration, as defined by ESPEN (the European Society for Clinical Nutrition and Metabolism) [6]

Dehydration: a shortage of water (fluid) in the body, caused by either insufficient intake of water (low-intake dehydration) or excessive loss of water (volume depletion) or combination of both

Low-intake dehydration: a shortage of water caused by low intake. Leads to loss of both intracellular and extracellular fluid, raises osmolality

Volume depletion: an excessive loss of water and salts caused by bleeding, fever, vomiting, diarrhoea or other causes. Leads to loss of extracellular fluid but not intracellular fluid, keeps osmolality normal or low 


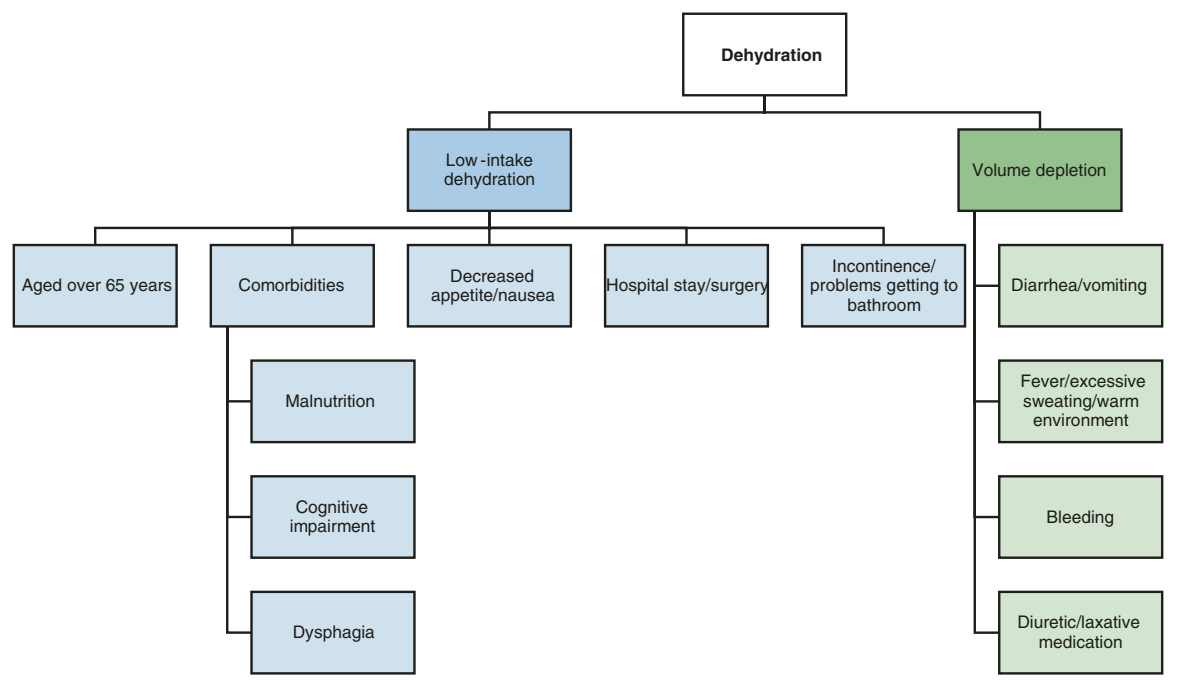

Fig. 7.1 Looking out for dehydration in older adults—red flags for nurses $[1,7,10]$

- Fluid intake history and abnormal losses

- Comorbidities (including malnutrition and refeeding syndrome)

- Clinical examination (pulse, blood pressure, capillary refill, jugular venous pressure, pulmonary or peripheral oedema, postural hypotension)

- Current medication

- Clinical measures (National Early Warning Score (NEWS), fluid balance charts and weight)

- Laboratory results (full blood count, urea, creatinine, electrolytes and osmolarity)

Systematically looking at the abovementioned factors, nurses can evaluate if older adults are at risk of dehydration/dehydrated and in turn consult with other healthcare providers, including but not limited to medical professionals and dietitians $[4,6]$.

\subsubsection{Assessing Low-Intake Dehydration}

In low-intake dehydration, the osmolality of fluid in the body is raised, as fluids are not replaced, whilst electrolytes are maintained [4, 6]. Based on this knowledge, the gold standard to both screen for and diagnose low-intake dehydration is direct measurement of serum/plasma osmolality (pOsm), using a threshold of $>300 \mathrm{mOsm} / \mathrm{kg}$. When direct measurement of osmolality is not available, the osmolarity equation $\left(\right.$ pOsmc $=1.86 \times\left(\mathrm{Na}^{+}+\mathrm{K}^{+}\right)+1.15 \times$ glucose + urea +14, all measured in $\left.\mathrm{mmol} / \mathrm{L}\right)$ should be used for diagnosis, using a threshold of $>295 \mathrm{mmol} / \mathrm{L}$ [11].

Traditionally a diagnosis of low-intake dehydration has been based on clinical assessment; however, in a recently published consensus on dehydration, Lacey et al. point out a lack of evidence for this approach in the literature [4]. Thus, using simple measures such as skin turgor, mouth dryness, weight change, urine colour or 
specific gravity to assess dehydration is not recommended, due to the inaccurate nature of these measures $[4,6]$. Measures shown to mildly correlate to low-intake dehydration, although having low diagnostic value, include expressions of fatigue, observed reduction in fluid oral intake and bioelectrical impedance analysis.

\subsubsection{Assessing Volume Depletion}

Irrespective of measured serum/plasma osmolality, all patients should be assessed for volume status $[4,5]$. According to NICE (National Institute for Health and Care Excellence) guidelines on intravenous fluid therapy in adults in hospital, using the following indicators of volume depletion to assess the need for intravenous fluid therapy is recommended:

- Systolic blood pressure $<100 \mathrm{mmHg}$

- Heart rate $>90$ beats per minute

- Capillary refill time $>2$ s/peripheries cold to touch

- Respiratory rate $>20$ breaths per minute

- National Early Warning Score (NEWS) $\geq 5$

- Passive leg raising, suggesting fluid responsiveness

Volume depletion following excessive blood loss can be assessed using postural pulse change from lying to standing of $\geq 30$ beats per minute or by observing severe dizziness, resulting in inability to stand $[4,6]$. Volume depletion in patients suffering from vomiting or diarrhoea can be assessed using the following set of signs (with $\geq 4$ out of 7 indicating depletion):

- Confusion

- Non-fluent speech

- Extremity weakness

- Dry mucous membranes

- Dry tongue

- Furrowed tongue

- Sunken eyes

\subsubsection{Prevention and Treatment of Dehydration}

\subsubsection{Low-Intake Dehydration}

Geriatric patients at risk or diagnosed with low-intake dehydration should be encouraged to increase fluid intake by offering drinks of different choices frequently, and fluid balance should be reassessed frequently [2, 4, 6]. For patients having difficulties with drinking, subcutaneous or intravenous fluids should be considered along with encouraging increased fluid intake. As for patients unable to drink, intravenous fluids should be considered. 
Important factors in increasing fluid intake include:

- Goal setting and policies by hospitals and geriatric wards regarding fluid intake.

- Staff awareness and support for drinking.

- Educating patients on the importance of drinking.

- Recording individual preferences for drinks.

- Proactive offering of preferred drinks to patients.

- Taking patients to the toilet as quickly as possible when needed.

- Considering physical and visual limitations of patients (e.g. sport bottles to prevent spilling or using bright-coloured cups for visibility).

- Assessment of swallowing, providing relevant treatment to patients with signs of dysphagia.

\subsubsection{Volume Depletion}

Whilst focusing on underlying causes and trying to minimise fluid loss, geriatric patients at risk or diagnosed with volume depletion should routinely receive isotonic fluids orally (via rehydration therapy), nasogastrically, subcutaneously or intravenously [4-6]. Aiming to replace lost water and electrolytes, patients with severe depletion should routinely receive intravenous fluid therapy immediately, whilst those with milder depletion should have their needs met orally or enterally when possible and appropriate.

\subsubsection{Fluid Overload}

Excessive fluid intake can result in fluid overload, a state where intake exceeds renal or cardiac capacity, possibly resulting in life-threatening hyponatremia, renal failure and/or heart failure [2, 5]. Main risk factors for fluid overload include decreased renal and/or cardiac function, surgery and receiving intravenous fluid therapy. Thus, fluid intake and output should be closely monitored, especially in patients receiving intravenous fluid therapy.

\subsubsection{Electrolyte Imbalances}

Imbalances in serum sodium levels are commonly diagnosed in geriatric and especially orthogeriatric patients and should be monitored $[5,12]$. Both hyponatremia (low sodium levels, $<135 \mathrm{mmol} / \mathrm{L}$ ) and hypernatremia (high sodium levels, $>145 \mathrm{mmol} / \mathrm{L}$ ) can have severe consequences and have been associated with falls, delirium, increased length of stay and increased 30-day mortality.

Serum potassium levels should be monitored in patients receiving intravenous fluid therapy, due to increased risk of either hypokalaemia $(<3.0 \mathrm{mmol} / \mathrm{L})$ or hyperkalaemia (>5.5 mmol/L) [5]. 


\subsection{Hospitalisation After Fall}

\subsubsection{Arriving at the Emergency Department}

Joe is an 84-year-old man who slipped when he was walking in his apartment this morning. He couldn't stand up, so his wife Julie called an ambulance. Physical examination shows his left leg to be shorter and externally rotated and he is in a lot of pain. Arriving at the ED, he is categorised as possibly having a hip fracture and therefore he is instructed to fast.

In many settings, including this case example hospital, older orthopaedic patients arriving at the emergency department (ED) after falling are fasted until diagnosis is established, in case an operation is needed [13].

As soon as diagnosis is established, assessment of fluid needs, fluid balance and the need of intravenous fluid therapy is needed; these should be guided by local procedural documentation considerate of recommendations and guidelines such as those described above. Any signs of volume depletion indicate that urgent fluid is needed, and recommendations highlight the importance of starting intravenous fluid therapy as soon as possible after arrival at the ED or possibly in the ambulance [3, $5,13]$. According to A Guide to Improving the Care of Fragility Fractures, it is critical to start early rehydration at the ED, using isotonic crystalloids [3]. Starting dehydration prevention at the beginning of the stay at the ED is a crucial component of treatment and has been shown to increase survival rates.

Joe has been at the ED for $2 h$ and has already gone to radiography, after which he was diagnosed with a hip fracture. Joe thinks he is lucky because he has a lovely nurse, Margareth, who has given him morphine for the pain. She has told him that he needs an operation to fix his fracture. Before Joe fell this morning, he hadn't eaten breakfast, and now Margareth has told him he can't eat before the operation and he will need intravenous fluid. However, due to Margareth having five other patients, she has not yet managed to put up his fluid.

\subsubsection{Preoperation Nursing at the Ward}

After a decision has been made according to admission and the patient is moved to a ward, fluid balance should be reassessed and appropriate treatment ensured [13]; again these should be informed by local processes considerate of recommendations and guidelines such as those described above (Sects. 7.3.1 and 7.3.2). When an operation is needed, knowing approximately at what time the operation will be is important as decreasing the fasting time of the patient as much as possible is recommended [13]. 
Joe doesn't feel so bad now that the morphine is working and he can even fall asleep from time to time. Margareth has told him that he will be moved to the orthopaedic ward, where she hands him over to the nurses and forgets to talk about his need for fluid therapy.

Geriatric patients are commonly dehydrated, and the risk of dehydration is increased during hospital stay $[3,6,13]$. Thus, when drinking is allowed, nurses and nursing staff should create an opportunity for the patient to drink, offering preferred drinks proactively at each contact with the patient. Generally, across many settings patients may have solids until $6 \mathrm{~h}$ before surgery and approved preoperative oral fluids until $2 \mathrm{~h}$ before surgery and then be placed nil by mouth until surgery $[13,14]$. However, fasting guidelines vary according to types of surgery performed, different patient cohorts and the patient's individual circumstances, and we recommend local interdisciplinary teams work together to implement procedures and processes to safely minimise fasting times.

The nurse who is now taking care of Joe also has a lot of tasks to do, but it suddenly crosses her mind why he doesn't have intravenous fluid. Joe hasn't called the nurse, as he doesn't feel so bad when lying completely still in bed. The nurse previously told him that the operation would be soon. Finally, the theatre call the ward and say that they are ready to operate on Joe. The nurse now hangs up intravenous fluid, but it's now been $8 \mathrm{~h}$ since he fell and almost 16 since he last ate or drank.

Correcting/preventing fluid imbalance, such as volume depletion and electrolyte disturbances that can affect comorbidities, is crucial before operation [5]. For this patient group, fluid overload is also a risk, possibly causing heart failure, peripheral oedema and hemodynamic imbalance, increasing the risk of delaying operation and/or complications post-operation.

\subsubsection{Post-operation Nursing at the Ward}

When a patient is moved from theatre to the ward, fluid balance should be reassessed and appropriate treatment ensured [15]; again this should be guided by locally developed, evidence-informed procedures and processes.

After operation, oral hydration and nutrition should be started as soon as possible, aiming to fulfil both fluid and nutritional needs [2, 4, 5, 15]. Regular reassessment of fluid balance should be provided during hospital stay. 
Case example: Joe's operation went well, but when he arrives back at the orthopaedic ward, he is having hallucinations. He is in delirium and refuses to drink because he thinks the nurses are trying to poison him. He then tries to take the IV catheter from his hand. At further examination Joe is also diagnosed with acute kidney injury, and his vital signs show low blood pressure and a high pulse.

Special considerations post-operation should be given to:

Delirium: a threatening risk factor for geriatric and orthogeriatric patients, who are at high risk of delirium as they are older than 65 years and some have current hip fracture, two of the major risk factors for delirium [13, 16] (Chap. 19). Interventions to prevent delirium are recommended, including prevention and treatment of dehydration and frequent assessment of fluid balance.

Acute kidney injury (AKI): a major complication, sometimes associated with dehydration [4, 5]. Risk factors for AKI include age higher than 65 years, having acute surgery (i.e. orthopaedic surgery) and use of NSAIDs medicine. Monitoring signs of oliguria is recommended (less than $0.5 \mathrm{~mL} / \mathrm{kg} / \mathrm{h}$ of urine in more than $6 \mathrm{~h}$, raised serum creatinine and $25 \%$ fall in glomerular filtration rate).

\subsubsection{Best Practice at the Ward}

Nurses and other staff members should follow recommendations and clinical guidelines regarding hydration, fluid balance and intravenous fluid therapy at all stages in hospital, including rehabilitation and at hospital discharge [2, 5, 15]. Preoperative carbohydrate drinks are considered to be harmless and have possible benefits on fluid balance; such drinks can be used up to $2 \mathrm{~h}$ before operation, thus possibly preventing dehydration, as well as allowing for early treatment, minimising the risk of adverse outcomes $[17,18]$.

Rewriting Joe's story in the best way, he would have been given an IVF in the ambulance the way to the hospital. Margareth, the ED nurse, would have screened for risk of dehydration, set up fasting clock to manage his fasting time and put up a fluid therapy plan for him as well as evaluated his fluid balance. The orthopaedic nurse would have checked the time plan in theatre for Joe and re-evaluated his fluid balance when he arrived at the ward and accordingly constructed a treatment plan, as well as given him a pre-op carbohydrate drink. Would this be the case, Joe may have been less likely to have developed delirium and avoided consequent preventable harm. 


\subsection{Summary}

All hospitalised geriatric patients should be screened and assessed for dehydration, and frequent reassessment of fluid balance is recommended. Dehydration can be defined into two subcategories, based on whether a shortage of water is caused by low intake or excessive loss. Offering preferred drinks frequently to patients in a proactive way is encouraged at all stages in hospital, and fasting time before operation should be minimised. Frequent reassessment of fluid balance is key to assessing the need for fluid intake and/or intravenous fluid therapy at all stages.

\section{Take-Home Points}

- Geriatric and orthogeriatric patients are at high risk of dehydration.

- Dehydration and fluid balance should be assessed frequently during hospital stay, allowing for early detection, treatment and possibly prevention.

- Evidence-based recommendations and guidelines for hydration, fluid intake and intravenous fluid therapy should be applied at all stages in hospital.

\section{References}

1. Volkert D, Beck AM, Cederholm T, Cruz-Jentoft A, Goisser S, Hooper L et al (2019) ESPEN guideline on clinical nutrition and hydration in geriatrics. Clin Nutr 38(1):10-47

2. Meehan AJ, Maher AB, Brent L, Copanitsanou P, Cross J, Kimber C et al (2019) The International Collaboration of Orthopaedic Nursing (ICON): best practice nursing care standards for older adults with fragility hip fracture. Int J Orthop Trauma Nurs 32:3-26

3. Mears SC, Kates SL (2015) A guide to improving the care of patients with fragility fractures, Edition 2. Geriatr Orthop Surg Rehabil 6(2):58-120

4. Lacey J, Corbett J, Forni L, Hooper L, Hughes F, Minto G et al (2019) A multidisciplinary consensus on dehydration: definitions, diagnostic methods and clinical implications. Ann Med 51(3-4):232-251

5. National Institute for Health and Care Excellence (2013) Intravenous fluid therapy in adults in hospital [CG174]. https://www.nice.org.uk/guidance/cg174

6. Volkert D, Beck AM, Cederholm T, Cruz-Jentoft A, Goisser S, Hooper L et al (2019) ESPEN guideline on clinical nutrition and hydration in geriatrics. Clin Nutr 38(1):10-47

7. Hooper L, Abdelhamid A, Attreed NJ, Campbell WW, Channell AM, Chassagne P et al (2015) Clinical symptoms, signs and tests for identification of impending and current water-loss dehydration in older people. Cochrane Database Syst Rev (4):CD009647

8. Edmonds CJ, Foglia E, Booth P, Fu CHY, Gardner M (2021) Dehydration in older people: a systematic review of the effects of dehydration on health outcomes, healthcare costs and cognitive performance. Arch Gerontol Geriatr 95:104380

9. EFSA Panel on Dietetic Products N (2010) Allergies. Scientific opinion on dietary reference values for water. EFSA J 8(3):1459

10. Thomas DR, Cote TR, Lawhorne L, Levenson SA, Rubenstein LZ, Smith DA et al (2008) Understanding clinical dehydration and its treatment. J Am Med Dir Assoc 9(5):292-301 
11. Hooper L, Abdelhamid A, Ali A, Bunn DK, Jennings A, John WG, Kerry S, Lindner G, Pfortmueller CA, Sjöstrand F, Walsh NP, Fairweather-Tait SJ, Potter JF, Hunter PR, Shepstone L (2015) Diagnostic accuracy of calculated serum osmolarity to predict dehydration in older people: adding value to pathology laboratory reports. BMJ Open. Oct 21;5(10):e008846. https://doi.org/10.1136/bmjopen-2015-008846. PMID: 26490100; PMCID: PMC4636668.

12. Madsen CM, Jantzen C, Lauritzen JB, Abrahamsen B, Jorgensen HL (2016) Hyponatremia and hypernatremia are associated with increased 30-day mortality in hip fracture patients. Osteoporos Int 27(1):397-404

13. Mohanty S, Rosenthal RA, Russell MM, Neuman MD, Ko CY, Esnaola NF (2016) Optimal perioperative management of the geriatric patient: a best practices guideline from the American College of Surgeons NSQIP and the American Geriatrics Society. J Am Coll Surg 222(5):930-947

14. Australian and New Zealand College of Anaesthetists (2017) Guideline on pre-anaesthesia consultation and patient preparation. https://www.anzca.edu.au/resources/professional-documents/ guidelines/ps07-guidelines-on-pre-anaesthesia-consultation-an

15. Powell-Tuck J, Gosling P, Lobo D, Allison S, Carlson G, Gore M, et al (2011) British consensus guidelines on intravenous fluid therapy for adult surgical patients. https://www.bapen.org. uk/pdfs/bapen_pubs/giftasup.pdf

16. National Institute for Health and Care Excellence (2010) Delirium: prevention, diagnosis and management [CG103]. https://www.nice.org.uk/guidance/cg103

17. Lobo DN, Gianotti L, Adiamah A, Barazzoni R, Deutz NEP, Dhatariya K et al (2020) Perioperative nutrition: recommendations from the ESPEN expert group. Clin Nutr (Edin, Scot) 39(11):3211-3227

18. Hellström PM, Samuelsson B, Al-Ani AN, Hedström M (2017) Normal gastric emptying time of a carbohydrate-rich drink in elderly patients with acute hip fracture: a pilot study. BMC Anesthesiol 17(1):23

\section{Recommended Reading}

Lacey J, Corbett J, Forni L, Hooper L, Hughes F, Minto G et al (2019) A multidisciplinary consensus on dehydration: definitions, diagnostic methods and clinical implications. Ann Med 51(3-4):232-251

National Institute for Health and Care Excellence (2013) Intravenous fluid therapy in adults in hospital [CG174]. https://www.nice.org.uk/guidance/cg174

Volkert D, Beck AM, Cederholm T, Cruz-Jentoft A, Goisser S, Hooper L et al (2019) ESPEN guideline on clinical nutrition and hydration in geriatrics. Clin Nutr (Edin, Scot) 38(1):10-47

Open Access This chapter is licensed under the terms of the Creative Commons Attribution 4.0 International License (http://creativecommons.org/licenses/by/4.0/), which permits use, sharing, adaptation, distribution and reproduction in any medium or format, as long as you give appropriate credit to the original author(s) and the source, provide a link to the Creative Commons license and indicate if changes were made.

The images or other third party material in this chapter are included in the chapter's Creative Commons license, unless indicated otherwise in a credit line to the material. If material is not included in the chapter's Creative Commons license and your intended use is not permitted by statutory regulation or exceeds the permitted use, you will need to obtain permission directly from the copyright holder.

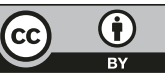

